\title{
Variations in TM6SF2, PCSK9 and PCSK7 genes and risk of hepatic steatosis after liver transplantation: a cross-sectional study
}

\author{
Ahad Eshraghian ${ }^{1}$, Elham Moasser2 ${ }^{2 *}$ Negar Azarpira ${ }^{2 *}$, Mohammad Reza Fattahi ${ }^{3}$, Saman Nikeghbalian ${ }^{1,2}$, \\ Seyed Ali Malek-Hosseini ${ }^{1,2}$ and Bita Geramizadeh ${ }^{2}$
}

\begin{abstract}
Background: Genetic abnormalities might have important role in pathogenesis of hepatic steatosis after liver transplantation. We aimed to investigate association between genetic variations in transmembrane 6 superfamily member 2 (TM6SF2) rs58542926, proprotein convertase subtilisin/kexin type 9 (PCSK9) rs505151 and proprotein convertase subtilisin/kexin type 7 (PCSK7) rs2277287 with hepatic steatosis in liver transplant recipients.

Methods: In a cross-sectional study, adult (> 18 years) liver transplant recipients who were referred for their routine post-transplant follow-up between June 2018 and September 2018 were included in the study. Hepatic steatosis in transplant recipients was assessed by controlled attenuation parameter (CAP). Polymerase chain reaction-restriction fragment length polymorphism (PCR-RFLP) was used to study TM6SF2 rs58542926, PCSK7 rs2277287 and PCSK9 rs505151 genotypes.

Results: 107 liver transplant recipients were included. There was no association between different genotypes of PCSK9 rs505151 and PCSK7 rs2277287 with hepatic steatosis in liver transplant recipients ( $P$ value $>0.05)$. The presence of TT genotype of TM6SF2 rs58542926 was higher in patients with hepatic steatosis measured by CAP after liver transplantation. In patients with moderate and severe hepatic steatosis (grade 2 and 3 steatosis), AG + GG genotypes of PCSK9 rs505151 were more prevalent than AA genotype (OR 8.667; 95\% Cl 1.841-40.879; $P$ value $=0.004)$ compared to patients with mild steatosis (grade 1). In multivariate regression model, AG + GG genotypes of PCSK9 rs505151 were associated with moderate and severe steatosis in liver transplant recipients (OR 5.747; 95\% Cl 1.086-30.303; $P$ value $=0.040$ )
\end{abstract}

Conclusions: Genetic variations in TM6SF2 rs58542926 and PCSK9 rs505151 might be associated with hepatic steatosis in liver transplant recipients.

Keywords: Liver transplant, Hepatic steatosis, Non-alcoholic fatty liver disease, Hepatic steatosis, Genetic variants

*Correspondence: Elhammoasser@yahoo.com; Azarpiran@sums.ac.ir

${ }^{2}$ Transplant Research Center, Shiraz University of Medical Sciences,

Research Tower, PO Box 71994-67985, Shiraz, Iran

Full list of author information is available at the end of the article

\section{Introduction}

Clinical outcomes of liver transplant recipients have been improved during recent years leading to increased survival of patients. In parallel, chronic adverse outcomes including metabolic abnormalities have been increased $[1,2]$. Hepatic steatosis might occur after liver transplantation with an estimated reported prevalence of $30-60 \%$ in different studies [3, 4]. Pathogenesis of hepatic 
steatosis after liver transplantation has not been well elucidated yet. We have recently demonstrated that alterations in serum adipokines and insulin resistance are main contributing factors in hepatic steatosis after liver transplantation [5]. Genetic susceptibility might also have important role in pathogenesis of non-alcoholic fatty liver disease (NAFLD) [6]. Patatin like phospholipase domain-containing 3 (PNPLA3) rs738409 polymorphism has been reported to be associated with hepatic steatosis, steatohepatitis and liver fibrosis in patients with NAFLD [7, 8]. Similarly, genetic predisposition might be a risk factor for hepatic steatosis in liver transplant recipients. It has been reported that liver transplant recipients with $G$ allele in position rs738409 of PNPLA3 were at increased risk for graft steatosis [9]. Transmembrane 6 superfamily member 2 (TM6SF2) is a gene encoding a transmembrane protein located in endoplasmic reticulum and Golgi apparatus and acts as a lipid transporter [10]. TM6SF2 rs58542926 variant was shown to be associated with hepatic steatosis independent of other metabolic risk factors [11]. It has been suggested that TM6SF2 rs58542926 variant is associated with hepatic steatosis via affecting glucose homeostasis, nutrient oxidation, postprandial lipoprotein and adipokine [12]. However, its impact on graft steatosis after liver transplantation is not clear. Proprotein convertase subtilisin/kexin type 9 (PCSK9) is an endoprotease synthesized in liver and is involved in clearance of low density lipoprotein (LDL) in hepatocytes [13]. Two recent studies have shown circulating PCSK9 was associated with hepatic steatosis [14] and PCSK9 rs11591147 loss of function mutation had protective effect against hepatic steatosis [15]. Proprotein convertase subtilisin/kexin type 7 (PCSK7) is another endoprotease from subtilisin-like proprotein convertase family that is involved in lipid metabolism [16]. Although, PCSK9 and PCSK7 can theoretically be associated with hepatic steatosis there is few clinical studies in this regard. We conducted this study to explore the association between genetic variations in TM6SF2 rs58542926, PCSK9 rs505151 and PCSK7 rs2277287 and hepatic steatosis in liver transplant recipients.

\section{Methods}

In a cross-sectional study, liver transplant recipients who had undergone liver transplantation from deceased donors at Shiraz Transplant Center, Shiraz, Iran, were included. Patients were referred for their routine posttransplant follow-up between June 2018 and September 2018. Patients were included if: (a) they were adult (>18 years), (b) at least 6 months had been passed from transplant surgery, (c) and did not have malignancy. We used tacrolimus based immunosuppressive regimen for all patients as maintenance of immunosuppression after liver transplantation. Patients with acute rejection episodes and those with serum aspartate aminotransferase and/or alanine aminotransferase levels higher than 5 times upper limit of normal range were excluded from the study. Clinical characteristics of patients including age, gender, underlying liver disease, post-transplant diabetes (PTDM), hyperlipidemia, hypertension and laboratory data were recorded at the time of study inclusion. Body mass index (BMI) was calculated using this formula: weight $(\mathrm{kg})$ divided by height $\left(\mathrm{m}^{2}\right)$ squared. Hepatic steatosis in transplant recipients was assessed by controlled attenuation parameter (CAP).

We used CAP measurement during vibration controlled transient elastography (VCTE) (FibroScan ${ }^{\circledR}$, Echosens, Paris, France) for estimation of hepatic steatosis in all liver transplant recipients. CAP measurement was done after an overnight fasting on the day of clinical visit and blood sampling. All CAP measurements were performed by one person with sufficient expertise with the procedure. CAP measurement was considered to be reliable if 10 valid successful acquisitions were obtained. CAP measurement was performed using $M$ probe in all patients. In obese patients, in whom use of $M$ probe was failed, XL probe was used for CAP measurement. CAP measurement was expressed in decibel per meter $(\mathrm{dB} / \mathrm{m})$ [17]. The cutoff scores for CAP estimation of hepatic steatosis were: $\geq 238 \mathrm{~dB} / \mathrm{m}$ and $<259 \mathrm{~dB} / \mathrm{m}$ for $\mathrm{S} 1$ (mild steaosis) (corresponding to $11-32 \%$ liver fat), $\geq 259 \mathrm{~dB} / \mathrm{m}$ and $<292 \mathrm{~dB} / \mathrm{m}$ for S2 (moderate steatosis) (33-65\% liver

Table 1 Primers used for TM6SF2, PCSK7, and PCSK9 polymorphisms typing

\begin{tabular}{|c|c|c|c|c|c|}
\hline SNP & Primer $\left(5^{\prime} \rightarrow 3^{\prime}\right)$ & $\begin{array}{l}\text { Annealing } \\
\text { temperature }\left({ }^{\circ} \mathrm{C}\right)\end{array}$ & Amplicon (bp) & Restriction enzyme & RFLP fragments (bp) \\
\hline TM6SF2-rs58542926 & $\begin{array}{l}\text { F: CCAGGTGTTGTCTCAAGTGG } \\
\text { R: CCAGGTGTTGTCTCAAGTGG }\end{array}$ & 60 & 247 & Нру1881 & $\begin{array}{l}\text { T allele: } 247 \\
\text { C allele: } 175+71\end{array}$ \\
\hline PCSK7-rs2277287 & $\begin{array}{l}\text { F: CATTGCCTAGGTATCCGGGT } \\
\text { R: GGGCTTCTCATGTGGCAATC }\end{array}$ & 60 & 321 & Pvull & $\begin{array}{l}\text { Tallele: } 321 \\
\text { Callele: } 226+95\end{array}$ \\
\hline PCSK9-rs505151 & $\begin{array}{l}\text { F: CACGGTTGTGTCCCAAATGG } \\
\text { R: GAGAGGGACAAGTCGGAACC }\end{array}$ & 60 & 440 & Eam 11041 (Earl) & $\begin{array}{l}\text { G allele: } 440 \\
\text { A allele: } 290+150\end{array}$ \\
\hline
\end{tabular}


Table 2 Baseline characteristics of patients in the study

\begin{tabular}{|c|c|}
\hline Age (years) & $43.21 \pm 12.99$ \\
\hline Sex (female/male), n (\%) & $47 / 60(43.9 / 56.1)$ \\
\hline BMI $\left(\mathrm{kg} / \mathrm{m}^{2}\right)$ & $24.92 \pm 5.17$ \\
\hline PTDM, n (\%) & $33(32.4)$ \\
\hline HTN, n (\%) & $26(26)$ \\
\hline HLP, n (\%) & $27(26.5)$ \\
\hline Rejection, n (\%) & $31(35.2)$ \\
\hline \multicolumn{2}{|c|}{ Etiology of liver disease (n, \%) } \\
\hline HBV & $19(17.7)$ \\
\hline $\mathrm{HCV}$ & $4(3.7)$ \\
\hline $\mathrm{NASH}$ & $14(13)$ \\
\hline $\mathrm{AlH}$ & $11(10.2)$ \\
\hline PSC & $21(19.6)$ \\
\hline Cryptogenic & $15(14)$ \\
\hline Others & $23(21.4)$ \\
\hline AST (IU/L) & $19(9-164)$ \\
\hline $\mathrm{ALT}(\mathrm{IU} / \mathrm{L})$ & $22.5(8-226)$ \\
\hline Alk.Phos (IU/L) & $200(44-800)$ \\
\hline Steatosis in TE $(n, \%)$ & $37(34.6)$ \\
\hline Mean CAP (dB/m) & $207.76 \pm 56.84$ \\
\hline \multicolumn{2}{|c|}{ TM6SF2 rs58542926 genotype (n, \%) } \\
\hline $\mathrm{CC}$ & $77(71.9)$ \\
\hline $\mathrm{CT}$ & $28(26.1)$ \\
\hline $\mathrm{TT}$ & $2(1.8)$ \\
\hline \multicolumn{2}{|c|}{ PCSK9 rs505151 genotype $(n, \%)$} \\
\hline AA & $54(50.4)$ \\
\hline$A G$ & $50(46.7)$ \\
\hline GG & $3(2.8)$ \\
\hline \multicolumn{2}{|c|}{ PCSK7 rs2277287 genotype (n, \%) } \\
\hline $\mathrm{CC}$ & $26(24.2)$ \\
\hline $\mathrm{CT}$ & $44(41.1)$ \\
\hline TT & 37 (34.5) \\
\hline
\end{tabular}

$B M /$ body mass index, PTDM post transplant diabetes mellitus, HLP hyperlipidemia, $H T N$ hypertension, $H B V$ hepatitis B virus, $H C V$ hepatitis $C$ virus, NASH non-alcoholic steatohepatitis, AlH autoimmune hepatitis, PSC primary sclerosing cholangitis, AST aspartate aminotransferase, ALT alanine aminotransferase, Alk.Phos alkaline phosphatase, TE transient elastography, TM6SF2 transmembrane 6 super family 2, PCSK9 proprotein convertase subtilisin/kexin type 9, PCSK7 proprotein convertase subtilisin/kexin type 7

fat) and $\geq 292 \mathrm{~dB} / \mathrm{m}$ for $\mathrm{S} 3$ (severe steatosis) ( $\geq 66 \%$ liver fat) [17].

\section{Genotyping}

Whole blood was collected from the liver transplant recipients in EDTA tubes and the genomic DNA was extracted from the peripheral blood leukocytes using high yield DNA purification Sinaclon Kit DNP ${ }^{\mathrm{TM}}$ protocol (SINACLON, Tehran, Iran) according to the manufacturer's instructions. The DNA extracted kit was placed in room temperature and all phases of DNA extraction were done under laminar hood. All instruments such as samplers and sampler tips, micro tubes, racks were sterilized by autoclave and $70 \%$ alcohol. Blood tubes were placed in room temperature. DNA was stored at $-20{ }^{\circ} \mathrm{C}$ until the time of usage.

The polymerase chain reaction (PCR) conditions for determining TM6SF2, PCSK9, and PCSK7 genotypes were set up. Briefly, the PCR protocol was performed using 300-500 ng of genomic DNA in total volume of $25 \mu \mathrm{L}, 200 \mu \mathrm{M}$ dNTPs, 10 pmol of each primer, $1.5 \mathrm{mM}$ $\mathrm{MgCl} 2$, and $1 \mathrm{U}$ Taq polymerase enzyme in a $10 \mathrm{mM}$ PCR buffer. The PCR protocol consisted of $5 \mathrm{~min}$ at $95^{\circ} \mathrm{C}, 35$ cycles of $1 \mathrm{~min}$ at $95^{\circ} \mathrm{C}, 1 \mathrm{~min}$ at $60{ }^{\circ} \mathrm{C}, 45 \mathrm{~s}$ at $72{ }^{\circ} \mathrm{C}$, and then $5 \mathrm{~min}$ at $72{ }^{\circ} \mathrm{C}$. Electrophoresis of $10 \mu \mathrm{L}$ of PCR products was performed on a $1.5 \%$ agarose gel as described before [18, 19]. Negative controls (tubes containing the PCR mixture, without the DNA template) were incubated in every run for prevention of contamination.

Polymerase chain reaction-restriction fragment length polymorphism (PCR-RFLP) was used to study TM6SF2 rs58542926, PCSK7 rs2277287 and PCSK9 rs505151 genotypes. To determine the TM6SF2 genotypes, the PCR product was digested using Hpy188I (New England Biolabs (NEB), UK) restriction enzyme at $37{ }^{\circ} \mathrm{C}$ for $16 \mathrm{~h}$. The rs2277287 polymorphism in the PCSK7 gene was studied using restriction endonuclease PvuII (New England Biolabs (NEB), UK) and the PCSK9-rs505151 variation was studied using Eam1104I (EarI) restriction enzyme (Thermo Fisher scientific, Inc, Waltham, MA, USA) (Table 1). The digested fragments were electrophoresed in $3 \%$ agarose gel and visualized with ultraviolet illumination. Furthermore, $10 \%$ of the PCR product underwent direct sequencing to verify the result of gel electrophoresis. There was complete agreement between the two methods.

\section{Statistical analysis}

Student's t-test, Mann-Whitney $U$ test and Chisquare test were used to analyze continuous and categorical variables. Data were presented using means \pm standard deviation for numeric variables, and percentages/counts for categorical variables. Logistic regression analysis was used to identify the independent variables and association of genetic variations with hepatic steatosis after liver transplantation. Statistically significant variables in univariate analysis were included in multivariate analysis. A $P$ value of $<0.05$ was considered statistically significant. Statistical analysis was performed with SPSS 20.0 (SPSS Inc.; Chicago, IL, USA). 


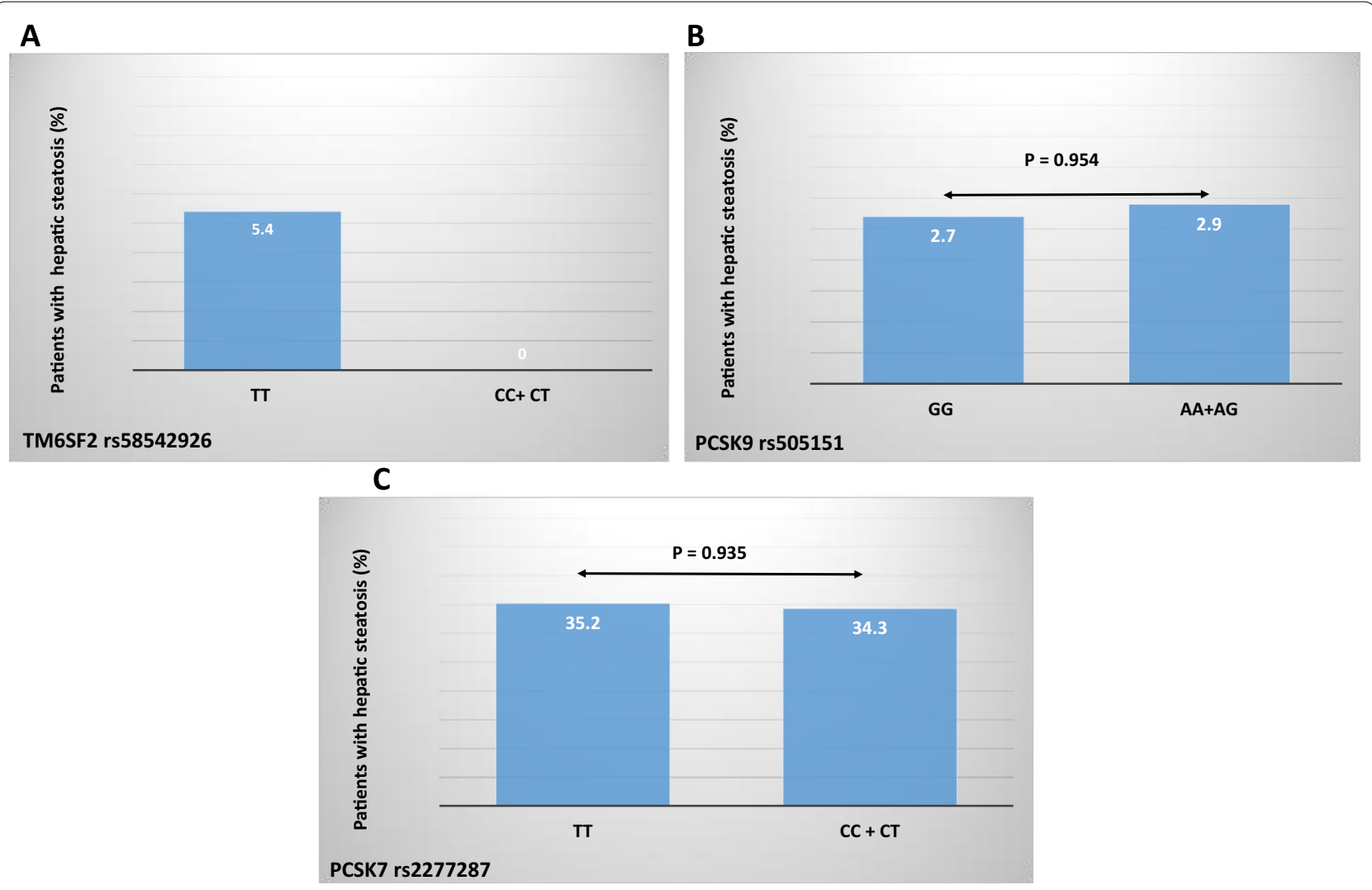

Fig. 1 Genetic variants of TM6SF2, PCSK9 and PCSK7 genes in liver transplant recipients with and without hepatic steatosis

\section{Results}

We evaluated 107 liver transplant recipients who had undergone liver transplantation for different etiologies of liver cirrhosis. Baseline characteristics of patients are outlined in Table 2. Mean time from transplant surgery to measurement of CAP was $41.16 \pm 35.85$ months. Thirty seven patients $(34.6 \%)$ were diagnosed to have different grades of hepatic steatosis in TE with a mean CAP of $207.76 \pm 56.84(\mathrm{~dB} / \mathrm{m})$. Seventeen patients $(45.9 \%)$ had grade 1 hepatic steatosis, 11 patients (29.7\%) had grade 2 hepatic steatosis and 9 patients $(24.3 \%)$ had grade 3 hepatic steatosis during CAP measurement.

The presence of steatosis in liver allografts after liver transplantation based on different variants of TM6SF2 rs58542926, PCSK9 rs505151 and PCSK7 rs2277287 genes are shown in Fig. 1. There was no association between different genotypes of PCSK9 rs505151 and PCSK7 rs2277287 with hepatic steatosis in liver transplant recipients $(P$ value $>0.05)$. The TT genotype of TM6SF2 rs58542926 was occurred in $5.4 \%$ of patients with hepatic steatosis and no patients without steaosis measured by CAP after liver transplantation. Univariate and multivariate analysis of risk factors for presence of hepatic steatosis after liver transplantation are outlined in Table 3. Higher age and BMI, post-transplant diabetes mellitus, post-transplant hyperlipidemia and hypertension were associated with hepatic steatosis in univariate analysis. In multivariate regression model, none of these variables were independent predictors of hepatic steatosis after liver transplantation among our study population (Table 3).

Patients were divided into those with mild steatosis (grade 1 steatosis) and those with moderate to severe steatosis (grade 2 and 3 steatosis). There were 17 patients $(45.9 \%)$ with mild steatosis (grade 1 ) and 20 patients (54\%) with moderate to severe steatosis (grade 2 and 3 steatosis). The presence of mild, moderate and severe steatosis in liver allografts based on different variants of TM6SF2 rs58542926, PCSK9 rs505151 and PCSK7 rs2277287 genes are shown in Fig. 1. There was no association between different genotypes of TM6SF2 rs58542926 and PCSK7 rs2277287 with moderate to severe hepatic steatosis in liver transplant recipients $(P$ value $>0.05$ ). In patients with moderate to severe hepatic steatosis, AG + GG genotypes of PCSK9 rs505151 were more prevalent compared to AA genotype (OR 8.667; 95\% CI 1.841-40.879; $P$ value $=0.004$ ) (Fig. 2). Other risk factors for presence of moderate to severe versus mild 
Table 3 Comparison of genetic variants and other risk factors in patients with and without hepatic steatosis after liver transplantation

\begin{tabular}{|c|c|c|c|c|c|c|}
\hline \multicolumn{4}{|l|}{ Univariate } & \multicolumn{3}{|c|}{ Multivariate analysis } \\
\hline & Steatosis (+) & Steatosis $(-)$ & $P$ value & OR & $95 \% \mathrm{Cl}$ & $P$ value \\
\hline Age (year) & $49.83 \pm 10.66$ & $39.71 \pm 12.81$ & $<0.001$ & 1.144 & $0.904-1.448$ & 0.253 \\
\hline Sex (male/female) & $23 / 14$ & $37 / 33$ & 0.356 & & & \\
\hline Height (cm) & $168.16 \pm 10.47$ & $166.73 \pm 9.36$ & 0.476 & & & \\
\hline Weight (kg) & $80.83 \pm 17.42$ & $63.82 \pm 11.58$ & $<0.001$ & & & \\
\hline BMI $\left(\mathrm{kg} / \mathrm{m}^{2}\right)$ & $28.59 \pm 5.70$ & $22.92 \pm 3.54$ & $<0.001$ & 1.074 & $0.768-1.503$ & 0.675 \\
\hline $\mathrm{HC}(\mathrm{cm})$ & $111.83 \pm 11.50$ & $97.29 \pm 8.14$ & $<0.001$ & 1.013 & $0.732-1.402$ & 0.938 \\
\hline WC (cm) & $104.49 \pm 12.04$ & $88.17 \pm 9.29$ & $<0.001$ & 1.114 & $0.827-1.501$ & 0.476 \\
\hline AST (IU/L) & $22.19 \pm 12$ & $26.41 \pm 25.71$ & 0.397 & & & \\
\hline ALT (IU/L) & $24.76 \pm 13.35$ & $32.14 \pm 34.70$ & 0.267 & & & \\
\hline Alk.Phos (IU/L) & $245.46 \pm 105.77$ & $212.35 \pm 185.23$ & 0.370 & & & \\
\hline PTDM & $61.1 \%$ & $16.7 \%$ & $<0.001$ & 0.168 & $0.008-3.647$ & 0.256 \\
\hline Post-transplant HLP & $47.2 \%$ & $15.2 \%$ & $<0.001$ & 1.222 & $0.085-17.64$ & 0.883 \\
\hline Post-transplant HTN & $38.9 \%$ & $18.8 \%$ & 0.028 & 0.324 & $0.017-6.318$ & 0.457 \\
\hline Rejection & $36.7 \%$ & $34.5 \%$ & 0.839 & & & \\
\hline Mean prednisolone dose (mg) & $8.26 \pm 3.87$ & $7.20 \pm 3.74$ & 0.379 & & & \\
\hline TM6SF2 genotype & & & & 1.237 & $0.083-18.473$ & 0.878 \\
\hline TT versus $C C+C T$ & $5.4 \%$ & 0 & & & & \\
\hline \multicolumn{7}{|l|}{ PCSK9 genotype } \\
\hline GG versus $A A+A G$ & $2.7 \%$ & $2.9 \%$ & 0.954 & & & \\
\hline \multicolumn{7}{|l|}{ PCSK7 genotype } \\
\hline TT versus CC+CT & $35.2 \%$ & $34.3 \%$ & 0.935 & & & \\
\hline
\end{tabular}

$B M I$ body mass index, AST aspartate aminotransferase, $A L T$ alanine aminotransferase, Alk.Phos alkaline phosphatase, $P T D M$ post-transplant diabetes mellitus, $H L P$ hyperlipidemia, HTN hypertension, WC waist circumference, HC hip circumference, TM6SF2 transmembrane 6 super family 2 , PCSK9 proprotein convertase subtilisin/ kexin type 9, PCSK7 proprotein convertase subtilisin/kexin type 7

hepatic steatosis after liver transplantation are outlined in Table 4. In multivariate regression model, AG+GG genotypes of PCSK9 rs505151 were associated with moderate to severe hepatic steatosis in liver transplant recipients (OR 5.747; 95\% CI 1.086-30.303; $P$ value $=0.040$ ).

\section{Discussion}

In this study, we investigated the association between variations in 3 candidate genes and hepatic steatosis after liver transplantation. Our results showed that frequency of TT genotype of TM6SF2 rs58542926 was higher in patients with hepatic steatosis than in patients without hepatic steatosis. In subgroup analysis, comparing patients with mild steatosis to patients with moderate to severe steatosis, AG + GG genotypes of PCSK9 rs505151 were associated with moderate and high grade steatosis in liver transplant recipients. Variations in PCSK7 rs2277287 gene was associated neither with presence nor degree of hepatic steatosis in liver transplant recipients.
TM6SF2 rs58542926 T allele and TT genotype are well known genetic risk factors for NAFLD, non-alcoholic steatohepatitis (NASH) and liver cirrhosis [20, 21]. This polymorphism changes glutamic acid to lysine amino acid causing down-regulation of TM6SF2 protein and increased deposition of triglycerides in hepatocytes [22]. On the other hand, overexpression of TM6SF2 protein causes decreased number and size of fat droplets in hepatocytes [23]. Previously, Mikova et al. showed that donor rs58542926 polymorphism in TM6SF2 gene was independently associated with graft steatosis and had additive effects on donor PNPLA3 [24]. However, a cross sectional study, with limited number of patients, failed to demonstrate association between genetic variations in recipient TM6SF2 rs58542926 and hepatic steatosis after liver transplantation [25]. In our findings, frequency of TM6SF2 rs58542926 TT genotype was higher in patients with hepatic steatosis after liver transplantation. 


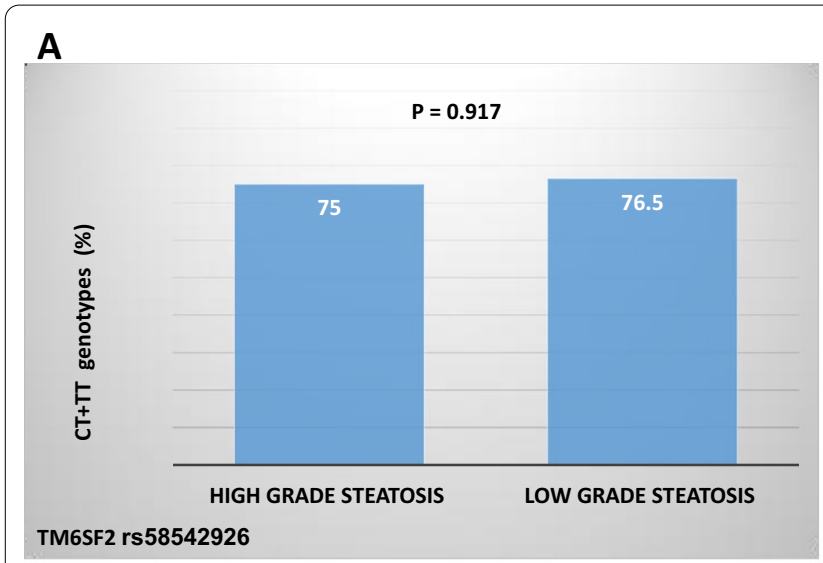

B
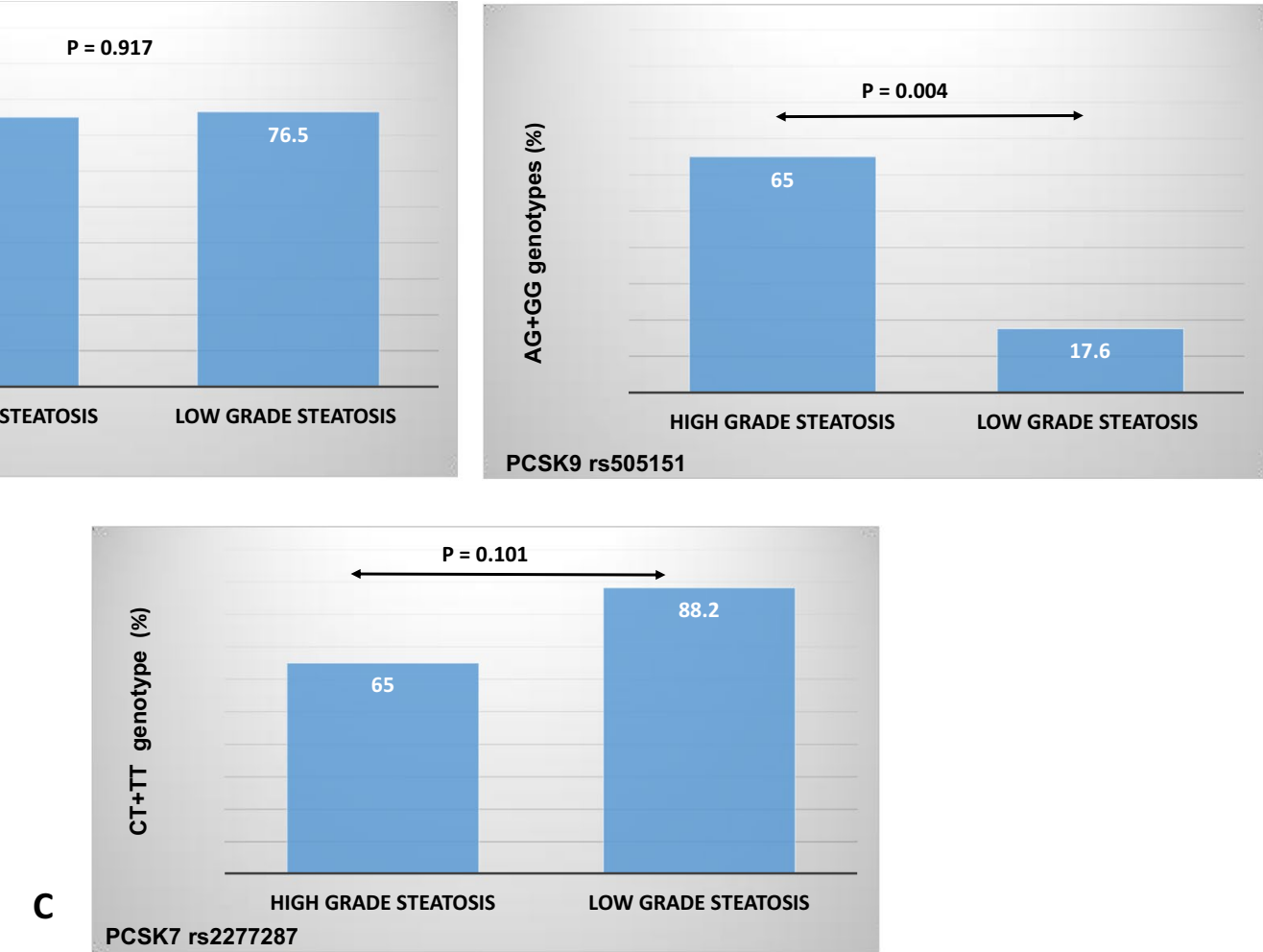

Fig. 2 Genetic variants of TM6SF2, PCSK9 and PCSK7 genes in liver transplant recipients with high grade (moderate to severe) steatosis and low grade (mild) steatosis

PCSK7 is a calcium-dependent serine endopeptidase located on chromosome 11 and is involved in the process of adipogenesis [16]. Ploso et al. showed that PCSK7 rs142953140 single nucleotide polymorphism was associated with serum lipid profile levels [26]. A genome wide association study (GWAS) showed that PCSK7 rs508487 single nucleotide polymorphism was associated with serum total cholesterol levels [27]. PCSK7 rs2277287 polymorphism has been recently reported to be associated with alterations in serum lipid profiles [28]. Dongiovanni et al. reported that PCSK7 rs236918 gene variations were associated with altered lipid profile and severe liver damage [29]. The role of PCSK $7 \mathrm{rs} 2277287$ gene in hepatic steatosis has not been previously investigated.

PCSK9 is synthesized and secreted by hepatocytes and has inhibitory effects on uptake of low density lipoproteins [30]. In clinical setting, PCSK9 rs505151 variants were associated with elevated LDL cholesterol in American Indians [13]. In a population of European ancestry, PCSK9 rs505151 polymorphism was reported in patients with hypercholesterolemia [31]. In a metaanalysis, increased triglycerides and LDL-cholesterol were associated with $\mathrm{G}$ allele of PCSK9 rs505151 [32]. It has been reported that circulating PCSK9 was associated with severity of hepatic steatosis, ballooning and fibrosis stage [33]. Protective effects of PCSK9 inhibitions against NAFLD and insulin resistance have been reported in recent studies [34]. Cariou et al. reported that plasma PCSK9 concentration was correlated with hepatic steatosis and hepatic insulin resistance in a population of young healthy volunteers fed with high fructose diet [35]. In our study, liver transplant recipients with AG + GG variants of PCSK9 rs505151 conferred an increased risk for higher degrees of hepatic steatosis as assessed by CAP.

Hepatic steatosis is an increasingly encountered problem after liver transplantation, however, pathogenesis is not clear. PNPLA3 rs738409 polymorphism is the most studied genetic variant for steatosis after liver transplantation and has been previously reported as a risk factor [36]. In this study, we tested liver transplant recipients with hepatic steatosis for genetic polymorphisms that had not been previously investigated. Our results revealed that TM6SF2 rs58542926 and PCSK9 rs505151 variants might be associated with hepatic steatosis after liver transplantation. Although our study reported novel clinical observations, it is a single center experience with limited number of patients. These findings should be replicated in future larger studies before 
Table 4 Comparison of genetic variants and other risk factors in patients with different grades of hepatic steatosis after liver transplantation

\begin{tabular}{|c|c|c|c|c|c|c|}
\hline \multicolumn{4}{|l|}{ Univariate } & \multicolumn{3}{|c|}{ Multivariate analysis } \\
\hline & $\begin{array}{l}\text { Moderate to severe } \\
\text { steatosis }\end{array}$ & Mild steatosis & $P$ value & OR & $95 \% \mathrm{Cl}$ & $P$ value \\
\hline Age (year) & $51.90 \pm 9.94$ & $47.41 \pm 11.27$ & 0.207 & & & \\
\hline Sex (male/female) & $10 / 10$ & $13 / 4$ & 0.098 & & & \\
\hline Height (cm) & $167.10 \pm 11.55$ & $169.41 \pm 9.23$ & 0.511 & & & \\
\hline Weight (kg) & $86 \pm 16.89$ & $74.76 \pm 16.47$ & 0.049 & & & \\
\hline $\mathrm{BMI}\left(\mathrm{kg} / \mathrm{m}^{2}\right)$ & $30.81 \pm 5.11$ & $25.99 \pm 5.38$ & 0.008 & 1.055 & $0.855-1.301$ & 0.620 \\
\hline WC $(\mathrm{cm})$ & $109.74 \pm 11.74$ & $99.29 \pm 9.95$ & 0.006 & 1.062 & $0.953-1.183$ & 0.276 \\
\hline AST (IU/L) & $21.62 \pm 9.4$ & $23.92 \pm 13.61$ & 0.590 & & & \\
\hline ALT (IU/L) & $24.43 \pm 11.76$ & $26.57 \pm 13.74$ & 0.650 & & & \\
\hline PTDM & $57.9 \%$ & $64.7 \%$ & 0.676 & & & \\
\hline Post-transplant HLP & $47.4 \%$ & $47.1 \%$ & 0.985 & & & \\
\hline Post-transplant HTN & $42.1 \%$ & $35.3 \%$ & 0.676 & & & \\
\hline Rejection & $36.7 \%$ & $34.5 \%$ & 0.839 & & & \\
\hline Mean prednisolone dose (mg) & $8.26 \pm 3.87$ & $7.20 \pm 3.74$ & 0.379 & & & \\
\hline \multicolumn{7}{|l|}{ TM6SF2 genotype } \\
\hline $\mathrm{CT}+\mathrm{TT}$ versus $\mathrm{CC}$ & $75 \%$ & $76.5 \%$ & 0.917 & & & \\
\hline \multicolumn{7}{|l|}{ PCSK9 genotype } \\
\hline$A G+G G$ versus $A A$ & $65 \%$ & $17.6 \%$ & 0.004 & 5.747 & $1.086-30.303$ & 0.040 \\
\hline \multicolumn{7}{|l|}{ PCSK7 genotype } \\
\hline$C T+T T$ versus $C C$ & $65 \%$ & $88.2 \%$ & 0.101 & & & \\
\hline
\end{tabular}

BMI body mass index, AST aspartate aminotransferase, ALT alanine aminotransferase, PTDM post-transplant diabetes mellitus, $H L P$ hyperlipidemia, $H T N$ hypertension, WC waist circumference, $H C$ hip circumference, TM6SF2 transmembrane 6 super family 2, PCSK9 proprotein convertase subtilisin/kexin type 9 , $P C S K 7$ proprotein convertase subtilisin/kexin type 7

any robust conclusion. On the other hand, genetic variations in donors might have impact on hepatic steatosis after liver transplantation and testing donors for specific genetic polymorphisms can be considered in future studies. Although the clinical importance of hepatic steatosis after liver transplantation is not still clear, it has been reported that advanced graft fibrosis was more prevalent in liver transplant recipients with graft steatosis after liver transplantation [37]. Therefore, liver transplant recipients with high risk genotypes might be considered for more intense follow-up and interventions for treatment.

\section{Acknowledgements}

Not applicable.

\section{Authors' contributions}

AE, EM, NA: study concept and design; acquisition of data; analysis and interpretation of data; drafting of the manuscript; critical revision of the manuscript for important intellectual content; statistical analysis. MRF, SN, BG, SAM: acquisition of data; analysis and interpretation of data; critical revision of the manuscript for important intellectual content. All authors read and approved the final manuscript.

\section{Funding}

The study was financially supported by a grant from Transplant Research Center, Shiraz University of Medical Sciences, Shiraz, Iran.

\section{Availability of data and materials}

The datasets generated and/or analysed during the current study are not publicly available due to keeping privacy of patients but are available from the corresponding author on reasonable request.

\section{Declarations}

Ethics approval and consent to participate

The study protocol was approved by the institutional review board of Shiraz Transplant Center, Abu-Ali Sina hospital. Written informed consents were obtained from each patient after explaining the study protocol including harms and benefits of the study during blood sampling and performing transient elastography. The study protocol was performed in accordance with the Helsinki Declaration as revised in Seoul 2008.

\section{Consent for publication}

Not applicable.

\section{Competing interests}

Authors declare that they have no conflict of interest.

\section{Author details}

${ }^{1}$ Shiraz Transplant Center, Abu-Ali Sina Hospital, Shiraz University of Medical Sciences, Shiraz, Iran. ${ }^{2}$ Transplant Research Center, Shiraz University of Medical Sciences, Research Tower, PO Box 71994-67985, Shiraz, Iran. ${ }^{3}$ Gastroenterohepatology Research Center, Shiraz University of Medical Sciences, Shiraz, Iran.

Received: 12 March 2021 Accepted: 22 November 2021

Published online: 07 December 2021 


\section{References}

1. Bodzin AS, Baker TB. Liver transplantation today: where we are now and where we are going. Liver Transpl. 2018;24(10):1470-5. https://doi.org/10. 1002/lt.25320.

2. Starzl TE, Fung JJ. Themes of liver transplantation. Hepatology 2010;51(6):1869-84. https://doi.org/10.1002/hep.23595.

3. Eshraghian A, Nikeghbalian S, Kazemi K, Shamsaeefar A, Geramizadeh B, Malek-Hosseini SA. Non-alcoholic fatty liver disease after liver transplantation in patients with non-alcoholic steatohepatitis and cryptogenic cirrhosis: the impact of pre-transplant graft steatosis. HPB (Oxford). 2020:22(4):521-8. https://doi.org/10.1016/j.hpb.2019.07.015.

4. Dumortier J, Giostra E, Belbouab S, et al. Non-alcoholic fatty liver disease in liver transplant recipients: another story of "seed and soil." Am J Gastroenterol. 2010;105:613-20.

5. Eshraghian A, Nikeghbalian S, Shamsaeefar A, Kazemi K, Fattahi MR, Malek-Hosseini SA. Hepatic steatosis and liver fat contents in liver transplant recipients are associated with serum adipokines and insulin resistance. Sci Rep. 2020;10(1):12701. https://doi.org/10.1038/ s41598-020-69571-1.

6. Sookoian S, Pirola CJ. Genetics of nonalcoholic fatty liver disease: from pathogenesis to therapeutics. Semin Liver Dis. 2019;39(2):124-40. https://doi.org/10.1055/s-0039-1679920.

7. Romeo S, Kozlitina J, Xing C, et al. Genetic variation in PNPLA3 confers susceptibility to nonalcoholic fatty liver disease. Nat Genet. 2008;40:1461-5.

8. Chalasani N, Guo X, Loomba R, et al. Genome-wide association study identifies variants associated with histologic features of nonalcoholic fatty liver disease. Gastroenterology. 2010;139:1567-1576. 1576.e1-6.

9. Finkenstedt A, Auer C, Glodny B, et al. Patatin-like phospholipase domain-containing protein 3 rs738409-G in recipients of liver transplants is a risk factor for graft steatosis. Clin Gastroenterol Hepatol. 2013;11(12):1667-72. https://doi.org/10.1016/j.cgh.2013.06.025.

10. Kozlitina J, Smagris E, Stender S, et al. Exome-wide association study identifies a TM6SF2 variant that confers susceptibility to nonalcoholic fatty liver disease. Nat Genet. 2014;46:352-6.

11. Sookoian S, Castaño GO, Scian R, et al. Genetic variation in transmembrane 6 superfamily member 2 and the risk of nonalcoholic fatty liver disease and histological disease severity. Hepatology. 2015;61(2):51525. https://doi.org/10.1002/hep.27556.

12. Musso G, Cipolla U, Cassader M, et al. TM6SF2 rs58542926 variant affects postprandial lipoprotein metabolism and glucose homeostasis in NAFLD. J Lipid Res. 2017;58(6):1221-9. https://doi.org/10.1194/jlr M075028.

13. Tsai CW, North $\mathrm{KE}$, Tin $\mathrm{A}$, et al. Both rare and common variants in PCSK9 influence plasma low-density lipoprotein cholesterol level in American Indians. J Clin Endocrinol Metab. 2015;100(2):E345-9. https://doi.org/ 10.1210/jc.2014-3340.

14. Paquette M, Gauthier D, Chamberland A, et al. Circulating PCSK9 is associated with liver biomarkers and hepatic steatosis. Clin Biochem. 2020;77:20-5.

15. Grimaudo S, Bartesaghi S, Rametta R, et al. PCSK9 rs11591147 R46L loss-of-function variant protects against liver damage in individuals with NAFLD. Liver Int. 2021;41(2):321-32.

16. Seidah NG, Hamelin J, Mamarbachi M, et al. CDNA structure, tissue distribution, and chromosomal localization of rat PC7, a novel mammalian proprotein convertase closest to yeast kexin-like proteinases. Proc Natl Acad Sci U S A. 1996:93:3388-93.

17. Sasso M, Beaugrand $M$, de Ledinghen $V$, et al. Controlled attenuation parameter (CAP): a novel VCTE ${ }^{\mathrm{TM}}$ guided ultrasonic attenuation measurement for the evaluation of hepatic steatosis: preliminary study and validation in a cohort of patients with chronic liver disease from various causes. Ultrasound Med Biol. 2010;36:1825-35.

18. Lorenz TC. Polymerase chain reaction: basic protocol plus troubleshooting and optimization strategies. J Vis Exp. 2012;63:3998.

19. Moasser E, Azarpira N, Shirazi B, Saadat M, Geramizadeh B. Genetic polymorphisms of glutathione-s-transferase M1 and T1 genes with risk of diabetic retinopathy in Iranian population. Iran J Basic Med Sci. 2014;17(5):351-6.
20. Liu YL, Reeves HL, Burt AD, et al. TM6SF2 rs58542926 influences hepatic fibrosis progression in patients with non-alcoholic fatty liver disease. Nat Commun. 2014;30(5):4309. https://doi.org/10.1038/ncomms5309.

21. Koo BK, Joo SK, Kim D, et al. Additive effects of PNPLA3 and TM6SF2 on the histological severity of non-alcoholic fatty liver disease. J Gastroenterol Hepatol. 2018;33(6):1277-85. https://doi.org/10.1111/jgh.14056.

22. Holmen $\mathrm{OL}$, Zhang $\mathrm{H}$, Fan $\mathrm{Y}$, et al. Systematic evaluation of coding variation identifies a candidate causal variant in TM6SF2 influencing total cholesterol and myocardial infarction risk. Nat Genet. 2014;46:345-51.

23. Mahdessian H, Taxiarchis A, Popov S, et al. TM6SF2 is a regulator of liver fat metabolism influencing triglyceride secretion and hepatic lipid droplet content. Proc Natl Acad Sci U S A. 2014;111:8913-8.

24. Míková I, Neřoldová M, Hubáček JA, et al. Donor PNPLA3 and TM6SF2 variant alleles confer additive risks for graft steatosis after liver transplantation. Transplantation. 2020;104(3):526-34. https://doi.org/10.1097/TP. 0000000000002876.

25. Liu ZT, Chen TC, Lu XX, et al. PNPLA3 I148M variant affects non-alcoholic fatty liver disease in liver transplant recipients. World J Gastroenterol. 2015;21(34):10054-6. https://doi.org/10.3748/wjg.v21.i34.10054.

26. Peloso GM, Auer PL, Bis JC, et al. Association of low-frequency and rare coding-sequence variants with blood lipids and coronary heart disease in 56,000 whites and blacks. Am J Hum Genet. 2014:94:223-32.

27. Kurano M, Tsukamoto K, Kamitsuji S, et al. Genome-wide association study of serum lipids confirms previously reported associations as well as new associations of common SNPs within PCSK7 gene with triglyceride. J Hum Genet. 2016;61:427433.

28. Zhang QH, Yin RX, et al. Association of the PCSK7 rs2277287 polymorphismand serum lipid levels in the Jing and Han populations. Int J Clin Exp Med. 2017;10(3):4986-5000.

29. Dongiovanni P, Meroni M, Baselli G, et al. PCSK7 gene variation bridges atherogenic dyslipidemia with hepatic inflammation in NAFLD patients. J Lipid Res. 2019;60(6):1144-53. https://doi.org/10.1194/jlr.P090449.

30. Gong Y, Ma Y, Ye Z, et al. Thyroid stimulating hormone exhibits the impact on LDLR/LDL-c via up-regulating hepatic PCSK9 expression. Metabolism. 2017;76:32-41. https://doi.org/10.1016/j.metabol.2017.07.006.

31. Chen SN, Ballantyne CM, Gotto AM Jr, Tan Y, Willerson JT, Marian AJ. A common pcsk9 haplotype, encompassing the e670g coding single nucleotide polymorphism, is a novel genetic marker for plasma low-density lipoprotein cholesterol levels and severity of coronary atherosclerosis. J Am Coll Cardiol. 2005:45:1611-9.

32. Qiu C, Zeng P, Li X, et al. What is the impact of PCSK9 rs505151 and rs11591147 polymorphisms on serum lipids level and cardiovascular risk: a meta-analysis. Lipids Health Dis. 2017;16(1):111. https://doi.org/10. 1186/s12944-017-0506-6

33. Ruscica $M$, Ferri $N$, Macchi $C$, et al. Liver fat accumulation is associated with circulating PCSK9. Ann Med. 2016;48(5):384-91. https://doi.org/10. 1080/07853890.2016.1188328.

34. Theocharidou E, Papademetriou M, Reklou A, Sachinidis A, Boutari C, Giouleme O. The role of PCSK9 in the pathogenesis of non-alcoholic fatty liver disease and the effect of PCSK9 inhibitors. Curr Pharm Des. 2018;24(31):3654-7. https://doi.org/10.2174/13816128246661810101 23127.

35. Cariou B, Langhi C, Le Bras M, et al. Plasma PCSK9 concentrations during an oral fat load and after short term high-fat, high-fat high-protein and high-fructose diets. Nutr Metab (London). 2013;10(1):4. https://doi.org/10 1186/1743-7075-10-4

36. Miyaaki H, Miuma S, Taura N, et al. PNPLA3 as a liver steatosis risk factor following living-donor liver transplantation for hepatitis C. Hepatol Res. 2018;48(3):E335-9. https://doi.org/10.1111/hepr.12920.

37. Yalamanchili K, Saadeh S, Klintmalm GB, Jennings LW, Davis GL. Nonalcoholic fatty liver disease after liver transplantation for cryptogenic cirrhosis or non-alcoholic fatty liver disease. Liver Transpl. 2010;16(4):431-9.

\section{Publisher's Note}

Springer Nature remains neutral with regard to jurisdictional claims in published maps and institutional affiliations. 